\title{
A development of the Enhanced Built-up and Bareness Index (EBBI) based on combination of multi-resolution Landsat 8 and Sentinel 2 MSI images
}

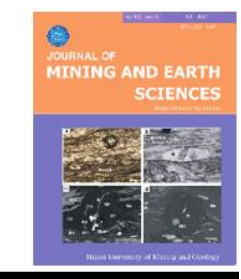

\author{
Hung Le Trinh ${ }^{1,}{ }^{*}$, Ha Thu Thi Le ${ }^{2}$, Loc Duc Le ${ }^{1}$, Long Thanh Nguyen ${ }^{3}$ \\ ${ }^{1}$ Military Technical Academy, Hanoi, Vietnam \\ ${ }^{2}$ Hanoi University of Mining and Geology, Vietnam \\ ${ }^{3}$ The Military Terrain Training Center, Lam Dong, Vietnam
}

\begin{tabular}{|c|c|}
\hline ARTICLE INFO & ABSTRACT \\
\hline $\begin{array}{l}\text { Article history: } \\
\text { Received } 15^{\text {th }} \text { Sept. } 2020 \\
\text { Revised } 23^{\text {rd }} \text { Dec. } 2020 \\
\text { Accepted } 21^{\text {st }} \text { Jan. } 2021\end{array}$ & $\begin{array}{l}\text { Classification of built-up land and bare land on remote sensing images is } \\
\text { a very difficult problem due to the complexity of the urban land cover. } \\
\text { Several urban indices have been proposed to improve the accuracy in } \\
\text { classifying urban land use/land cover from optical satellite imagery. This }\end{array}$ \\
\hline $\begin{array}{l}\text { Keywords: } \\
\text { Bare land, } \\
\text { Built-up land, } \\
\text { Multi-resolution images, } \\
\text { Remote sensing, } \\
\text { Urbanization. }\end{array}$ & $\begin{array}{l}\text { paper presents an development of the EBBI (Enhanced Built-up and } \\
\text { Bareness Index) index based on the combination of Landsat } 8 \text { and Sentinel } \\
2 \text { multi-resolution satellite imagery. Near infrared band (band 8a), short } \\
\text { wave infrared band (band 11) of Sentinel } 2 \text { MSI image and thermal } \\
\text { infrared band (band 10) Landsat } 8 \text { image were used to calculate EBBI } \\
\text { index. The results obtained show that the combination of Landsat } 8 \text { and } \\
\text { Sentinel } 2 \text { satellite images improves the spatial resolution of EBBI index } \\
\text { image, thereby improving the accuracy of classification of bare land and } \\
\text { built-up land by about 5\% compared with the case using only Landsat } 8 \\
\text { images. }\end{array}$ \\
\hline
\end{tabular}

Copyright (C) 2021 Hanoi University of Mining and Geology. All rights reserved.

${ }^{*}$ Corresponding author

E - mail: trinhlehung@lqdtu.edu.vn

DOI: 10.46326/JMES.2021.62(1).01 


\title{
Tạp chí Khoa học Kỹ thuật Mỏ - Địa chất
}

Trang điện tî̉: http://tapchi.humg.edu.vn

\section{Phát triển chỉ số đất đô thị EBBI (Enhanced Built-up and Bareness Index) trên cơ sở kết hợp ảnh vệ tinh đa độ phân giải Landsat 8 và Sentinel 2 MSI}

\author{
Trịnh Lê Hùng ${ }^{1,{ }^{*}}$, Lê Thị Thu Hà ${ }^{2}$, Lê Đức Lộc ${ }^{1}$, Nguyễn Thanh Long ${ }^{3}$ \\ ${ }^{1}$ Học viện Kỹ thuật Quân sự, Hà Nội, Việt Nam \\ 2 Trường Đại học Mỏ Địa chất, Hà Nội, Việt Nam \\ 3 Trung tâm Đào tạo Nghiệp vụ Địa hình Quân sự, Lâm Đồng, Việt Nam
}

\begin{abstract}
THÔNG TIN BÀI BÁO TÓM TẮT
Quá trình:

Nhậ $n$ bài $15 / 9 / 2020$

Sửa xong 23/12/2020

Chấp nhận đăng 21/01/2021

Tù̀ khóa:

Ảnh vệtinh đa độ phân giải

Đất trống,

Đất xây dựng,

Đô thị hoa,

Viễn thám.

Phân loại đất xây dựng và đất trống khu vực đô thị trên ảnh viễn thám là môt vấn đề rất khó khăn do sự phức tạp của lớp phủ bề mặt. Nhiều chỉ số đất đồ thị đã được đề xuất nhằm nâng cao độ chính xác khi phân loại sử dụng đất/lớp phủ trên ảnh vệ tinh quang học. Kênh cận hồng ngoại (8a), kênh hồng ngoại sóng ngắn (kênh 11), ảnh Sentinel 2 MSI cùng kênh hồng ngoại nhiệt (kênh 10), ảnh Landsat 8 được sử dụng để tính chỉ số EBBI. Bài báo này trình bày một phát triển của chỉ số EBBI (Enhanced Built-up and Bareness Index) trên cơ sở kết hợp ảnh vệ tinh đa độ phân giải Landsat 8 và Sentinel 2. Kết quả nhận được cho thấy, việc kết hợp ảnh vệ tinh Landsat 8 và Sentinel 2 giúp nâng cao độ phân giải không gian của ảnh chỉ số EBBI, tù̀ đó nâng cao độ chính xác khi phân loại đất trông và đất xây dựng lên khoảng $5 \%$ so với phương án chỉ sử dụng ảnh Landsat 8.
\end{abstract}

C 2021 Trường Đại học Mỏ - Địa chất. Tất cả các quyền được bảo đảm.

\section{Mở đầu}

Phân loại lớp phủ/sử dụng đất khu vực đô thị từ ảnh viễn thám là một vấn đề được nhiều nhà khoa học quan tâm nghiên cứu. Ban đầu, các phương pháp phân loại có giám sát và không giám sát được sử dụng để thành lập bản đồ hiện trạng sử dụng đất/lớp phủ khu vực đô thị (Xu, 2002;

\footnotetext{
*Tác giả liên hệ

E - mail: trinhlehung@lqdtu.edu.vn DOI: 10.46326/JMES.2021.62(1).01
}

Masek và nnk., 2000; Guindon và nnk., 2004). Tuy vậy, do lớp phủ bề mặt đô thị rất phức tạp, mỗi pixel trên ảnh viễn thám có thể bao gồm nhiều loại đối tượng lớp phủ khác nhau dẫn đến độ chính xác khi phân loại đất xây dựng, đất trống không cao.

Từ thập niên cuối thế kỷ XX, nhiều nhà khoa học đã sử dụng các chỉ số (Index) xác định từ ảnh viễn thám quang học nhằm nâng cao khả năng giải đoán các loại hình sử dụng đất/lớp phủ khu vực đô thị. Có thể kể đến các chỉ số như Urban index (UI) (Kawamura và nnk., 1996); Index based Built-up Index (IBI) (Xu, 2008); Normalized Difference Built-up Index (NDBI) (Zha và nnk., 
2003; Li và nnk., 2017); Normalized Difference Bareness Index (NDBaI) (Zhao và Chen, 2005); Bare soil index (BI) (Rikimaru và Miyatake, 1997); New Built-up Index (NBI) (Jieli và nnk., 2010); Modified Normalized Difference Impervious Surface Index (MNDISI) (Sun và nnk., 2017). Ở Việt Nam, một số nghiên cứu cũng đã sử dụng các chỉ số đất đô thị trong phân loại đất xây dựng và đất trống như Nguyễn Hoàng Khánh Linh (2011), Nguyễn Thúy Hạnh (2019). Nghiên cứu của Nguyễn Thúy Hạnh cho thấy, với trường hợp tại thủ đô Hà Nội, độ chính xác khi phân loại đất xây dựng bằng các chỉ số NDBI, IBI, EBBI có sự chênh lệch không lớn.

Chỉ số EBBI (Enhanced Build - up and Bareness Index) được As-syakur và cộng sự đề xuất trên cơ sở kết hợp các kênh hồng ngoại và hồng ngoại nhiệt ảnh vệ tinh Landsat nhằm theo dõi biến động đất đô thị ở Bali (Indonesia) (Assyakur và nnk., 2012). Trong nghiên cứu này, các tác giả đã chứng minh rằng chỉ số EBBI cho phép phân loại đất trống và đất xây dựng với độ chính xác cao hơn so với một số chỉ số đất đô thị khác như IBI, UI,... Một số nghiên cứu gần đây (Rasul và nnk., 2018; Bramhe và nnk., 2018; Sekertekin và nnk., 2018) cũng đã sử dụng chỉ số EBBI trong phân loại sử dụng đất/lớp phủ ở một số đô thị lớn.

Do độ phân giải không gian của ảnh Landsat 8 không cao (30 m ở các kênh đa phổ, 100 m ở kênh hồng ngoại nhiệt), việc sử dụng chỉ số EBBI xác định từ ảnh Landsat 8 trong phân loại đất trống và đất xây dựng gặp rất nhiều khó khăn, nhất là với những khu vực có diện tích nhỏ. Một số nghiên cứu gần đây ở Việt Nam đã chứng minh rằng: việc tích hợp ảnh vệ tinh Landsat 8 và Sentinel 2 MSI chụp sát thời điểm với nhau cho phép nâng cao độ phân giải không gian của nhiệt độ bề mặt (Trịnh Lê Hùng, 2018) hay chỉ số NDBaI (Trịnh Lê Hùng, 2020). Hơn nữa, việc lựa chọn các ảnh Landsat 8 và Sentinel 2 MSI chụp sát thời điểm với nhau giúp giảm thiểu ảnh hưởng của việc thay đổi lớp phủ bề mặt đến kết quả phân loại. Có thể nói, với cặp ảnh Landsat 8 và Sentinel 2 MSI chụp sát thời điểm với nhau (cùng ngày hoặc cách nhau 1 ngày), sự thay đổi của các đối tượng bề mặt tại khu vực đô thị là gần như không đáng kể.

Bài báo này trình bày một phát triển của chỉ số EBBI trên cơ sở tích hợp ảnh vệ tinh đa độ phân giải Landsat 8 và Sentinel 2. Kênh hồng ngoại nhiệt (kênh 10), ảnh Landsat 8 và kênh đỏ (kênh 8a), kênh hồng ngoại sóng ngắn (kênh 11) ảnh Sentinel 2 MSI được sử dụng để tính chỉ số EBBI. Điều này giúp nâng cao độ phân giải không gian của chỉ số EBBI lên $20 \mathrm{~m}$ so với $30 \mathrm{~m}$ nếu xác định từ riêng ảnh Landsat 8.

\section{Dũ̃ liệu và phương pháp nghiên cứu}

\subsection{Dũ̃ liệu}

Trong nghiên cứu này, ảnh Sentinel $2 \mathrm{~A}$ chụp ngày $01 / 10 / 2019$ (Hình $1 \mathrm{a}$ ) và Landsat 8 chụp ngày 30/9/2019 (Hình 1b) khu vực quận Long Biên và huyện Gia Lâm (Thành phố Hà Nội) được sử dụng để tính chỉ số EBBI. Đây là một trong những khu vực ở thủ đô Hà Nội có tốc độ đô thị hóa nhanh trong những năm gần đây. 02 ảnh được lựa chọn chụp gần thời điểm với nhau để giảm thiểu những khác biệt do sự khác nhau trong thời gian chụp ảnh gây nên.

So sánh đặc điểm các kênh ảnh vệ tinh Sentinel 2 MSI và Landsat 8 được trình bày trên Hình 2 (Kaab và nnk., 2016). Từ phân tích đặc

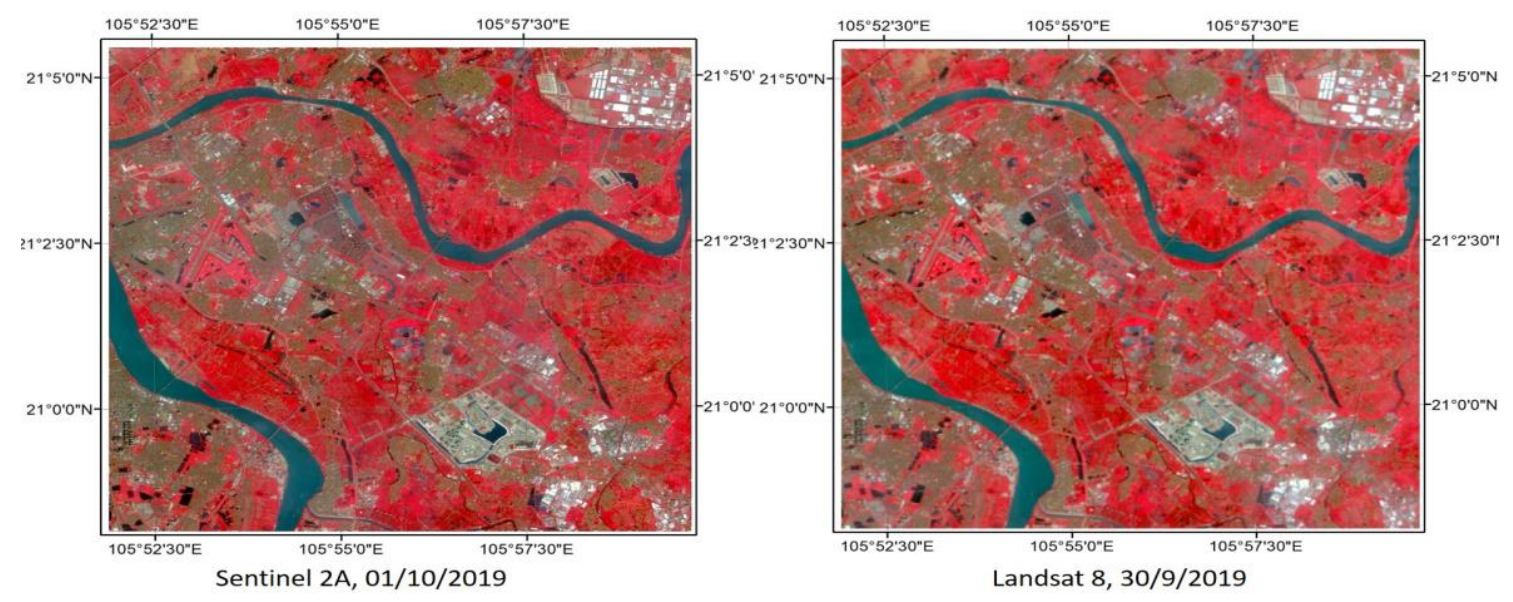

Hình 1. Ảnh vệ tinh Sentinel $2 A$ và Landsat 8 khu vực nghiên cúu. 
điểm các kênh phổ ảnh vệ tinh Landsat 8 và Sentinel 2 MSI cho thấy, kênh 5 (cận hồng ngoại) ảnh Landsat $8(0,845 \div 0,885 \mu \mathrm{m})$ và kênh 8 a ảnh Sentinel 2 MSI $(0,848 \div 0,881 \mu \mathrm{m})$ có bước sóng tương đồng nhau. Điều này cũng tương tự như với kênh 6 ảnh Landsat $8(1,560 \div 1,660 \mu \mathrm{m})$ và kênh 11 ảnh Sentinel 2 MSI $(1,539 \div 1,681 \mu \mathrm{m})$. Như vậy, có thể thay thế kênh 5 và kênh 6 ảnh Landsat 8 bằng các kênh $8 \mathrm{a}$ và 11 ảnh Sentinel 2 MSI chụp sát thời điểm với nhau khi tính chỉ số EBBI.

\subsection{Phương pháp nghiên cứu}

Chỉ số EBBI do Assyakur và nnk. (2012) đề xuất được xác định từ ảnh vệ tinh Landsat 8 như sau:

$$
E B B I=\frac{\text { Band } 6-\text { Band } 5}{10 \sqrt{\text { Band } 6+\text { Band } 10}}
$$

Trong đó: Band5, Band6, Band10 - các kênh cận hồng ngoại, hồng ngoại giữa và hồng ngoại nhiệt ảnh vệ tinh Landsat 8.

Chỉ số EBBI xác định trên cơ sở kết hợp ảnh vệ tinh đa độ phân giải Landsat 8 và Sentinel 2 MSI (ký hiệu là chỉ số iEBBI) theo công thức sau:

$$
=\frac{\text { Sentinel } 2_{\text {Band } 11}-\text { Sentinel } 2_{\text {Band } 8 a}}{10 \sqrt{\text { Sentinel } 2_{\text {Band } 11}+\text { Landsat } 8_{\text {Band } 10}}}
$$

Phương pháp phân ngưỡng được sử dụng để tách đất xây dựng và đất trống trên ảnh chỉ số iEBBI. Các giá trị ngưỡng này được lựa chọn bằng phương pháp chuyên gia trên cơ sở phân tích lược đồ histogram của ảnh chỉ số iEBBI. Quy trình phân loại đất xây dựng và đất trống khu vực đô thị bằng chỉ số iEBBI trên cơ sở kết hợp ảnh vệ tinh đa độ phân giải Sentinel 2 và Landsat 8 được mô tả trên Hình 3.

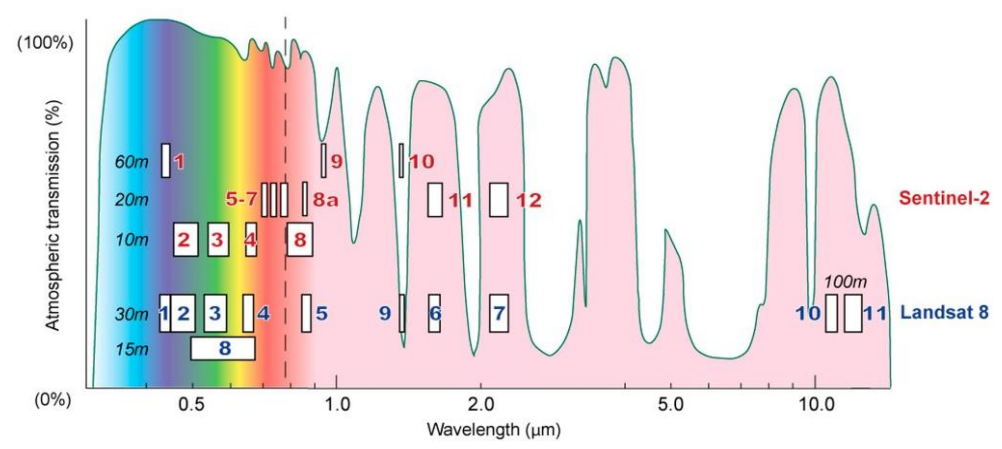

Hình 2. So sánh đặc điểm các kênh ảnh vệ tinh Sentinel 2 và Landsat 8 (Kaab và nnk., 2016).

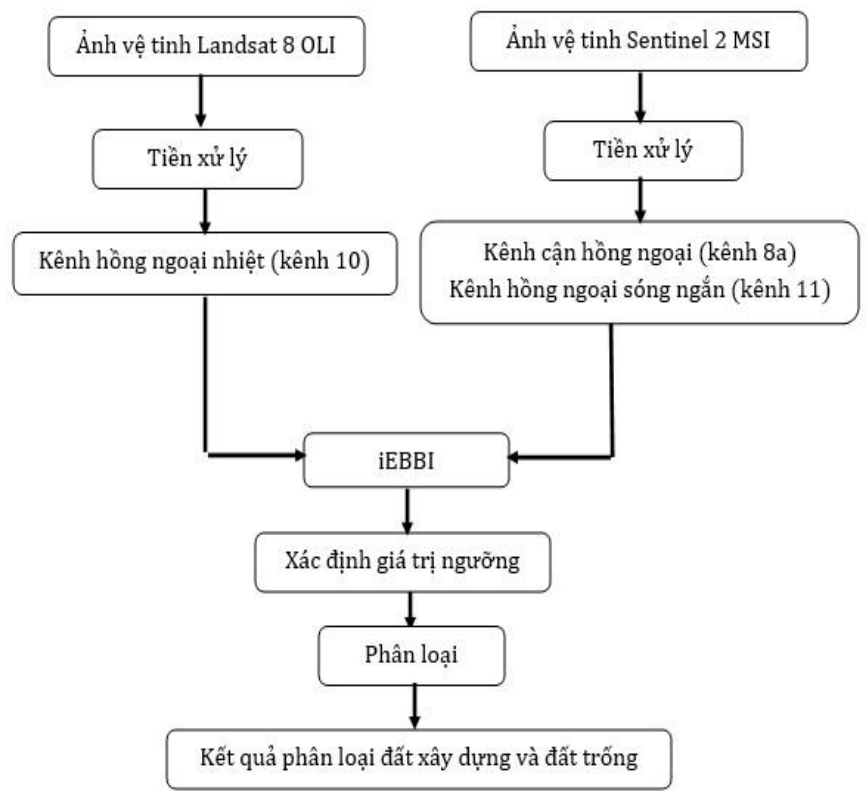

Hình 3. Quy trình phân loại đất xây dựng và đất trống khu vực đô thị từ chỉ số EBBI xác định trên cơ sở kết hợp ảnh vệ tinh Sentinel 2 MSI và Landsat 8. 


\section{Kết quả và thảo luận}

Để tính chỉ số EBBI từ ảnh vệ tinh Sentinel 2 MSI và Landsat 8 , nghiên cứu này xây dựng module phần mềm bằng ngôn ngữ lập trình Matlab. Ngôn ngữ Matlab này tỏ ra đặc biệt hiệu quả khi làm việc với các dữ liệu dạng ma trận, do vậy rất phù hợp khi xử lý ảnh viễn thám. Giao diện chương trình được thể hiện trên Hình 4.

Module phần mềm cung cấp khả năng mở, xử lý và hiển thị ảnh vệ tinh ở định dạng TIFF, trong đó bao gồm các công cụ chính như View (đọc và hiển thị ảnh), Urban indices (tính các chỉ số đất đô thị),... Ngoài chỉ số EBBI, trong module phần mềm cũng cung cấp công cụ tính các chỉ số đất đô thị thông dụng khác như NDBI, NDBaI, IBI. Hình 5 minh họa giao diện công cụ tính chỉ số iEBBI trên cơ sở kết hợp ảnh vệ tinh Sentinel 2 MSI và Landsat 8 , trong đó đầu vào bao gồm kênh cận hồng ngoại (kênh 8a), hồng ngoại sóng ngắn (kênh 11) ảnh Sentinel 2 MSI và kênh hồng ngoại nhiệt (kênh 10) ảnh Landsat 8.

Kết quả xác định chỉ số iEBBI trên cơ sở kết hợp ảnh vệ tinh đa độ phân giải Sentinel 2 MSI và Landsat 8 được thể hiện trên Hình 6 (a). Ngoài ra, để so sánh, đánh giá, trong nghiên cứu cũng tiến hành xác định chỉ số EBBI từ ảnh vệ tinh

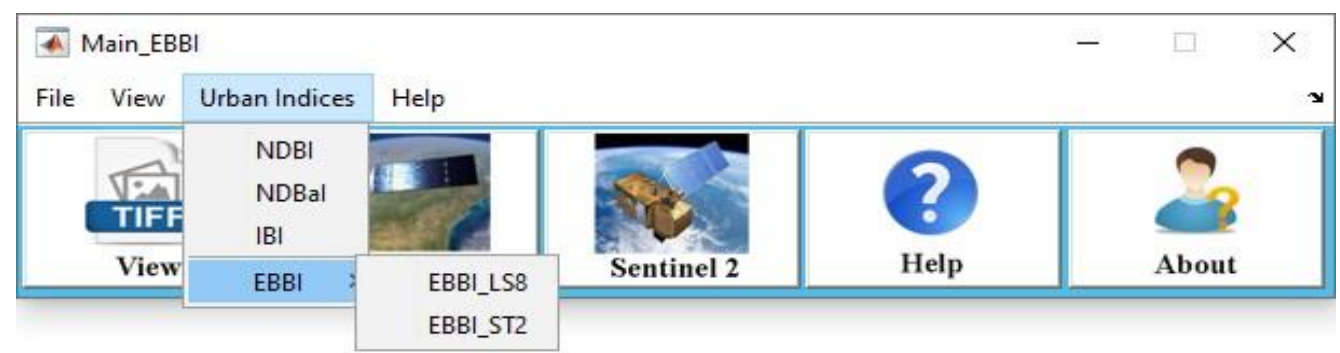

Hình 4. Giao diện module phần mềm xác định chỉ số EBBI.

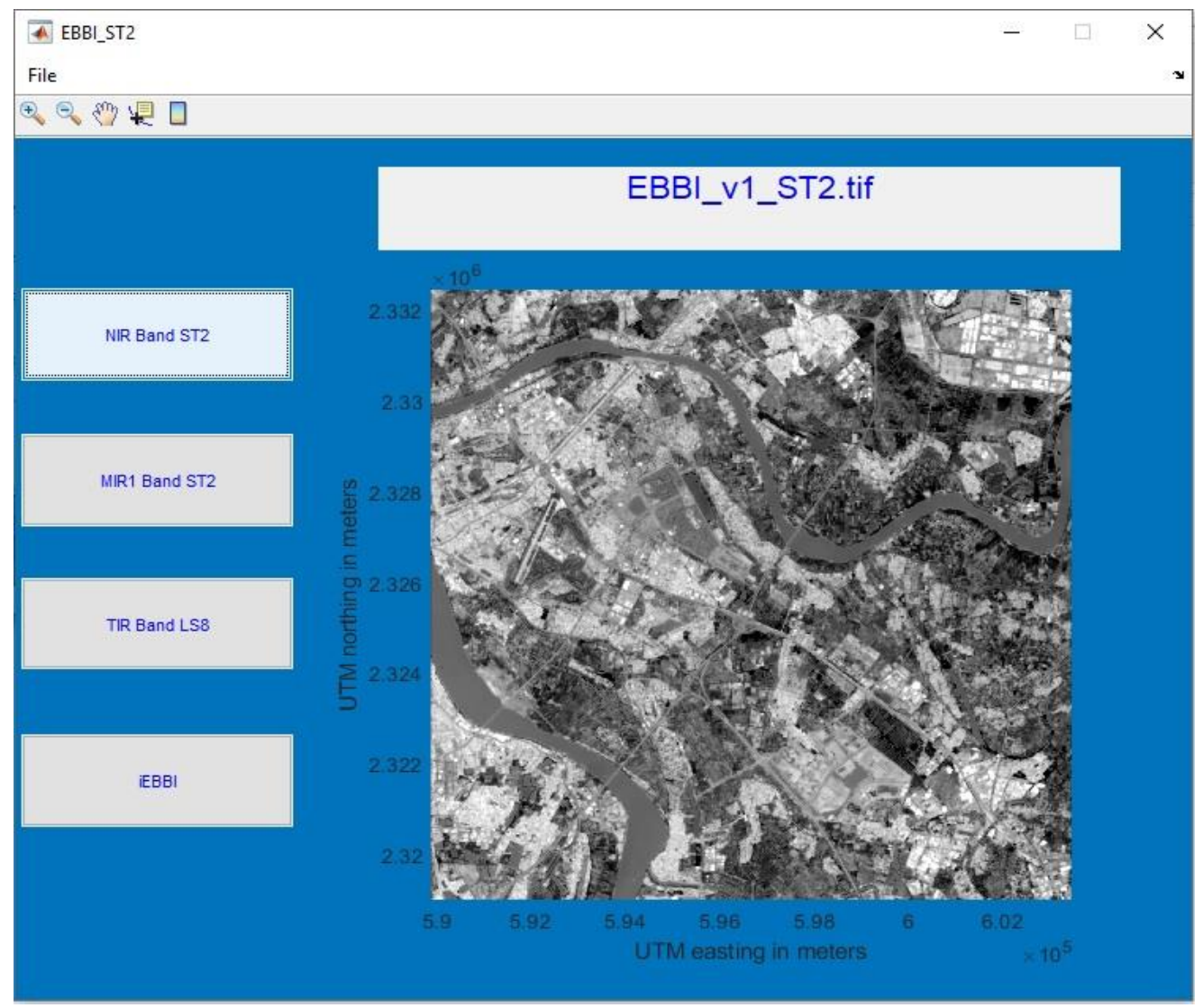

Hình 5. Công cụ tính chỉ sô iEBBI tù̀ ảnh vệ tinh Sentinel 2 MSI và Landsat 8. 
Landsat 8 (Hình 6b). Trên ảnh chỉ số EBBI, đất xây dựng có màu trắng sáng, đất trống có màu xám và các đối tượng lớp phủ khác có màu tối. Độ phân giải không gian của chỉ số iEBBI khi kết hợp sử dụng ảnh Sentinel 2 MSI và Landsat 8 đạt $20 \mathrm{~m}$ so với $30 \mathrm{~m}$ khi chỉ sử dụng ảnh vệ tinh Landsat 8.

Kết quả xác định chỉ số iEBBI trên cơ sở kết hợp ảnh vệ tinh đa độ phân giải Sentinel 2 MSI và Landsat 8 được thể hiện trên Hình 6 (a). Ngoài ra, để so sánh, đánh giá, trong nghiên cứu cũng tiến hành xác định chỉ số EBBI từ ảnh vệ tinh Landsat 8 (Hình 6b). Trên ảnh chỉ số EBBI, đất xây dựng có màu trắng sáng, đất trống có màu xám và các đối tượng lớp phủ khác có màu tối. Độ phân giải không gian của chỉ số iEBBI khi kết hợp sử dụng ảnh Sentinel 2 MSI và Landsat 8 đạt $20 \mathrm{~m}$ so với $30 \mathrm{~m}$ khi chỉ sử dụng ảnh vệ tinh Landsat 8 .

Phương pháp phân ngưỡng được sử dụng để chiết tách thông tin đất xây dựng, đất trống từ chỉ số EBBI. Đối với chỉ số iEBBI xác định từ việc kết hợp ảnh Sentinel 2 MSI và Landsat 8, đất xây dựng có giá trị lớn hơn 0,105 , trong khi đất trống có giá trị nằm trong khoảng $-0,024 \div 0,105$. Đối với phương án sử dụng chỉ số EBBI xác định từ ảnh
Landsat 8, đất xây dựng có giá trị lớn hơn -0,449; đất trống có giá trị nằm trong khoảng từ $-0,959 \div$ -0,449 (Bảng 1). Kết quả phân loại đất xây dựng, đất trống khu vực nghiên cứu từ chỉ số iEBBI và EBBI được thể hiện trên Hình 7.

Để đánh giá độ chính xác kết quả phân loại đất xây dựng và đất trống từ chỉ số EBBI, nghiên cứu này sử dụng 250 điểm kiểm tra ngẫu nhiên lấy từ kết quả phân loại, sau đó so sánh với ảnh vệ tinh độ phân giải cao thu thập từ Google Earth (Hình 8). Kết quả cho thấy, khi phân loại đất xây dựng và đất trống từ chỉ số iEBBI bằng cách kết hợp ảnh vệ tinh Landsat 8 và Sentinel 2 MSI, độ chính xác tổng thể đạt $91,2 \%$ và $89,6 \%$, chỉ số Kappa đạt 0,901 và 0,866 . Đối với phương án chỉ sử dụng ảnh vệ tinh Landsat 8, giá trị độ chính xác tổng thể khi phân loại đất xây dựng, đất trống đạt tương ứng $86,4 \%$ và $84,0 \%$; chỉ số Kappa đạt 0,849 và 0,812 . Như vậy, phương án sử dụng chỉ số iEBBI trên cơ sở kết hợp ảnh vệ tinh đa độ phân giải Sentinel 2 MSI và Landsat 8 giúp cải thiện độ chính xác khi phân loại đất xây dựng, đất trống lên khoảng $5 \%$ so với phương án chỉ sử dụng ảnh vệ tinh Landsat 8. Giá trị chỉ số Kappa cũng có sự gia tăng đáng kể.

Bảng 1. Kết quả phân loại đất xây dựng, đất trống từ chỉ số EBBI.

\begin{tabular}{|c|c|c|c|c|c|c|c|c|}
\hline \multirow{2}{*}{ TT } & \multirow{2}{*}{ Chỉ số } & \multirow{2}{*}{$\begin{array}{c}\text { Dữ liệu sư } \\
\text { dụng }\end{array}$} & \multicolumn{2}{|c|}{$\begin{array}{c}\text { Giá trị ngưỡng } \\
\text { dựng }\end{array}$} & Đất trống & \multicolumn{4}{|c|}{ Độ chính xác tổng thề chính xác } & \multicolumn{2}{|c|}{ Chỉ số Kappa } \\
\cline { 6 - 9 } & & & Đất xây dựng & Đất trống & Đất xây dựng & Đất trống \\
\hline 1 & iEBBI & $\begin{array}{c}\text { Sentinel 2 }+ \\
\text { Landsat 8 }\end{array}$ & $>0,105$ & $-0,024 \div 0,105$ & $91,2 \%$ & $89,6 \%$ & 0,901 & 0,886 \\
\hline 2 & EBBI & Landsat 8 & $>-0,449$ & $-0,959 \div-0,449$ & $86,4 \%$ & $84,0 \%$ & 0,849 & 0,812 \\
\hline
\end{tabular}

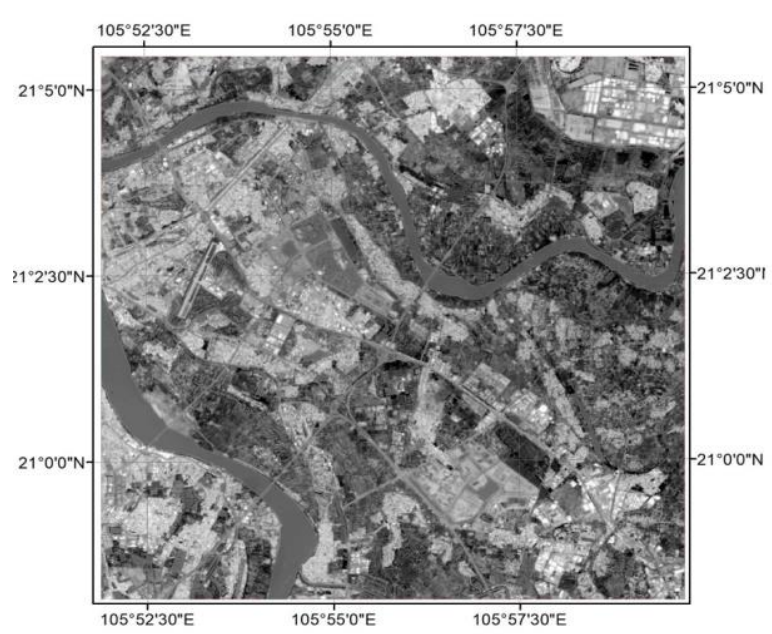

a)

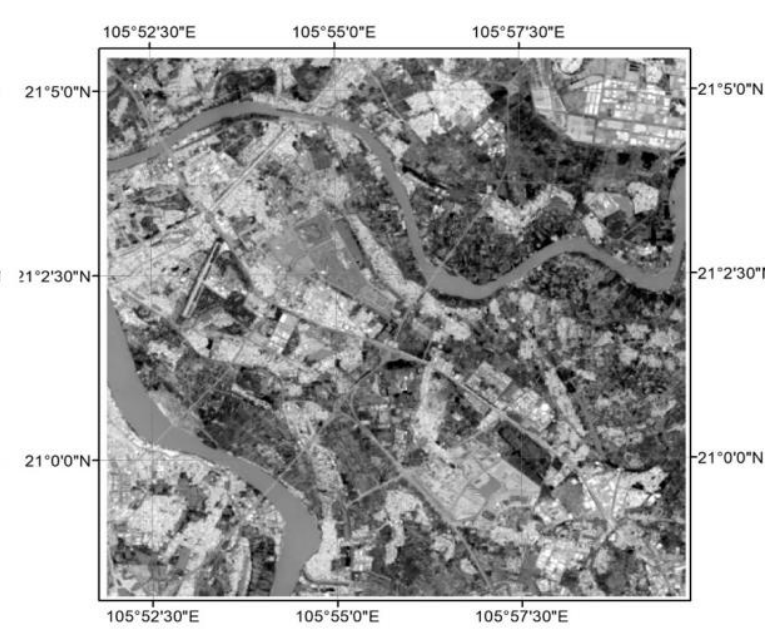

b)

Hình 6. Kết quả tính chỉ số EBBI trên cơ sở kết hợp ảnh vệ tinh Sentinel $2 A$ và Landsat 8 (a), chỉ sử dụng ảnh Landsat 8 (b). 


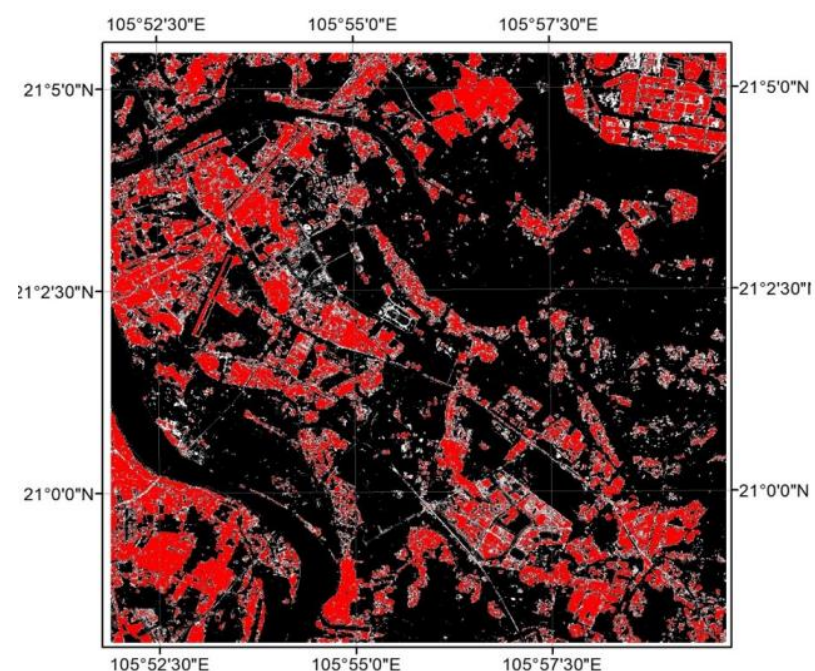

a)

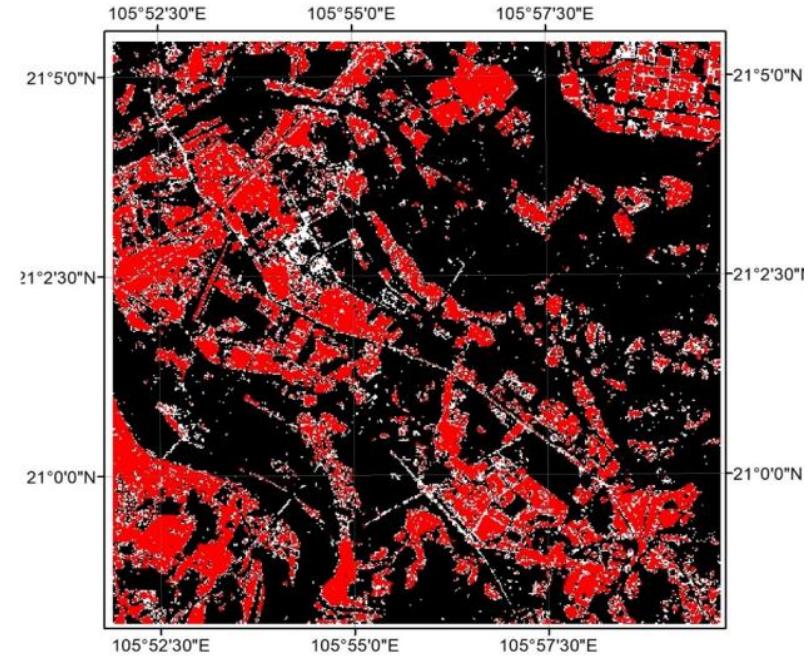

b)

Hình 7. Kết quả phân loại đất xây dựng và đất trống khu vực đô thị bằng chỉ số iEBBI (a) và EBBI (b).

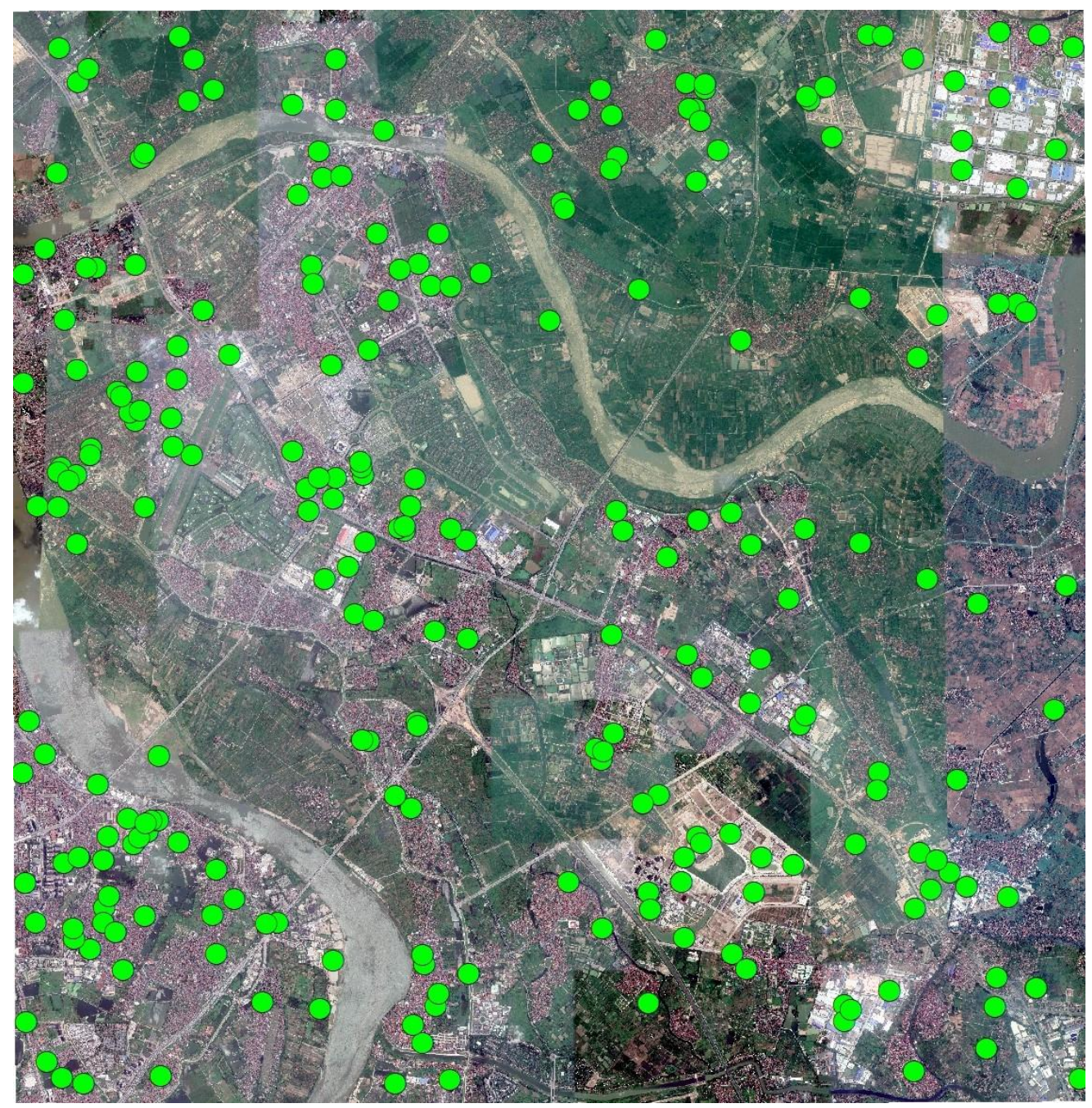

Hình 8. Phân bố các điểm lấy mẫu ngẫu nhiên trong đánh giá độ chính xác tù̀ ảnh Google Earth. 


\section{Kết luận}

Ảnh vệ tinh đa độ phân giải Sentinel 2 MSI và Landsat 8 với những đặc điểm tương đồng về dải phổ, thời gian cập nhật ngắn, được cung cấp hoàn toàn miễn phí có thể kết hợp sử dụng trong phân loại lớp phủ/sử dụng đất khu vực đô thị. Từ kết quả phân loại đất xây dựng, đất trống khu vực Hà Nội cho thấy rằng: việc sử dụng 02 cảnh ảnh Sentinel 2 MSI và Landsat chụp sát thời điểm với nhau để tính chỉ số EBBI cho phép nâng cao độ chính xác khi phân loại đất xây dựng, đất trống khu vực đô thị lên khoảng 5\%. Hơn nữa, độ phân giải không gian của chỉ số EBBI khi sử dụng kết hợp 2 loại dữ liệu viễn thám này cũng được cải thiện lên $20 \mathrm{~m}$ so với $30 \mathrm{~m}$ khi chỉ sử dụng ảnh Landsat 8. Kết quả nhận được trong nghiên cứu cũng cung cấp cơ sở khoa học góp phần nâng cao hiệu quả sử dụng các dữ liệu viễn thám miễn phí trong đánh giá, giám sát các thành phần tài nguyên, môi trường.

\section{Đóng góp của các tác giả}

Trịnh Lê Hùng, Lê Đức Lộc: lên ý tưởng; Trịnh Lê Hùng: viết bản thảo bài báo; Lê Thị Thu Hà, Lê Đức Lộc: đánh giá và chỉnh sửa.

\section{Tài liệu tham khảo}

As-syakur Abd. R., Adnyana I. W., Arthana I. W., Nuarsa I. W. (2012). Enhanced built - up and bareness index (EBBI) for mapping built - up and bare land in an urban area. Remote Sensing, 4, 2957-2970.

Bramhe V., Ghosh S., Garg P. (2018). Extraction of built-up area by combining textural features and spectral indices from Landsat 8 multispectral image. The International Archives of the Photogrammetry. Remote Sensing and Spatial Information Sciences, 42(5), 727-733.

Guindon B., Zhang Y., Dillabaugh C. (2004). Landsat urban mapping based on a combined spectral-spatial methodology. Remote Sensing of Environment, 92(2), 218 - 232.

Jieli C., Liu Y., Manchun L., Chenglei S. (2010). Extract residential areas automatically by $\mathrm{New}$ Built-up Index. The 18th International Conference on Geoinformatics: GIScience in
Change, Geoinformatics 2010, Peking University, Beijing, China, June, 18-20.

Kaab A., Winsvold S. H., Altena B., Nuth C., Nagler T., Wuite J. (2016). Glacier remote sensing using Sentinel 2. Part I: Radiometric and geometric performance and application to ice velocity. Remote Sensing, 8(7), 598, https://doi.org/10. 3390/rs8070598.

Kawamura M., Jayamana S., Tsujiko Y. (1996). Relation between social and environmental conditions in Colombo Sri Lanka and the urban index estimated by satellite remote sensing data. International Archives of the Photogrammetry. Remote Sensing and Spatial Information Sciences, 31(Part B7), 321-326.

Li H., Wang C., Zhong C., Su A., Xiong C., Wang J., Liu J. (2017). Mapping urban bare land automatically from Landsat imagery with a simple index. Remote Sensing, 9(3), 249, 1-15.

Masek J. G., Lindsay F. E., Goward S. N. (2000). Dynamics of urban growth in the Washington DC metropolitan area, 1973 - 1996 from Landsat observations. International Journal of Remote Sensing, 21(18), 3473-3486.

Nguyễn Hoàng Khá nh Linh. (2011). Thành lập tự động bản đồ phân bố đất đô thị bằng chỉ số IBI từ ảnh Landsat TM: Trường hợp nghiên cứu tại thành phố Huế - tỉnh Thừa Thiên Huế. Tuyển tập hội thảo Ứng dụng GIS toàn quốc. 205 - 12.

Nguyễn Thị Thúy Hạnh. (2019). Nghiên cứu chỉ số đô thị trong chiết tách đất trống và đất xay dựng khu vực Ha Nội từ ảnh vệ tinh Landsat 8. Tạp chí Khoa học Kỹ thuật Mỏ-Địa chất, 60(4), 82-86.

Rasul A., Balzter H., Faqe Ibrahim G., Hameed H., Wheeler J., Adamu B., Ibrahim S., Najmaddin P. (2018). Applying built-up and bare soil indicies from Landsat 8 to cities in dry climates. Land, 7, 81, 1-13.

Rikimaru A., Miyatake S. (1997). Development of Forest Canopy Density Mapping and Monitoring Model using Indices of Vegetation, Bare soil and Shadow. In Proceeding of the 18th Asian Conference on Remote Sensing (ACRS), Kuala Lumpur, Malaysia, 20-25 October 1997, p. 3. 
Sekertekin A., Abdikan S., Marangoz A. (2018). The acquisition of impervious surface area from Landsat 8 satellite sensor data using urban indices: a comparative analysis. Environmental Monitoring and Assessment, 190(7), 381, 1-13.

Sun Z., Wang C., Guo H., Shang R. (2017). A Modified Normalized Difference Impervious Surface Index (MNDISI) for automatic urban mapping from Landsat imagery. Remote Sensing, 9(9), 942, https://doi.org/10.3390/ rs9090942.

Trịnh Lê Hùng. (2018). Kết hợp ảnh vệ tinh Landsat 8 và Sentinel 2 trong nâng caoddooj phân giải nhiệt độ bề mặt. Tạp chí Khoa học Đại học Quốc gia Hà Nội. Chuyên san Các khoa học và Môi trường, 34(2), 54-63.

Trịnh Le Hung. (2020). Phân loại đất trống đô thị bằng chỉ số NDBaI trên cơ sở kết hợp ảnh vệ tinh đa độ phân giải Sentinel 2 MSI va Landsat 8. Tạp chí Khoa học Đại học Quốc gia Hà Nội, chuyên san Các Khoa học Trái đất và Môi trường, 36(2), 68-78.
Xu H. Q. (2002). Spatial expansion of urban/town in Fuqing and its driving analysis. Remote Sensing Technology and Application, 17, 8692.

Xu H.Q. (2007). Extraction of urban built - up land features from Landsat imagery using a thematic oriented index combination technique. Photogrammetric Engineering \& Remote Sensing, 73(12), 1381 -1391.

Xu H.Q. (2008). A new index for delineating builtup land features in satellite imagery. International Journal of Remote Sensing, 29, 4269 - 4276.

Zha Y., Gao J., Ni S. (2003). Use of normalized difference built-up index in automatically mapping urban areas from TM imagery. International Journal of Remote Sensing, 24(3), 583 - 594.

Zhao H., Chen X. (2005). Use of Normalized Difference Bareness Index in quickly mapping bare areas from TM/ETM+. International Geoscience and Remote Sensing Symposium (IGARSS) 3, 1666-1668, DOI: 10.1109/IGARSS. 2005.1526319 